\title{
Nanobubbles around plasmonic nanoparticles: Thermodynamic analysis
}

\author{
Julien Lombard, Thierry Biben, and Samy Merabia \\ Institut Lumière Matière, UMR5306 Université Lyon 1-CNRS, Université de Lyon, 69622 Villeurbanne cedex, France
}

(Received 8 August 2014; published 9 April 2015)

\begin{abstract}
We describe the dynamics of vapor nanobubbles in water, on the basis of simulations of a hydrodynamics phase-field model. This situation is relevant to recent experiments, where a water nanobubble is generated around a nanoparticle immersed in water, and heated by an intense laser pulse. We emphasize the importance of nanoscale effects in the dynamics of the nanobubble. We first analyze the evolution of the temperature inside the bubble. We show that the temperature drops by hundredths of kelvins in a few picoseconds, just after nanobubble formation. This is the result of the huge drop of the thermal boundary conductance between the nanoparticle and the fluid accompanying vaporization. Subsequently, the temperature inside the vapor is almost homogeneous and the temperature gradient is concentrated in the liquid, whose thermodynamic state locally follows the saturation line. We discuss also the evolution of the pressure inside the vapor nanobubble. We show that nanobubble generation is accompanied by a pressure wave propagating in the liquid at a velocity close to the liquid speed of sound. The internal pressure inside the vapor just after its formation largely exceeds Laplace pressure and quickly relaxes as a result of the damping generated by the viscous forces. All these considerations shed light on the thermodynamics of the nanobubbles generated experimentally.
\end{abstract}

DOI: 10.1103/PhysRevE.91.043007

PACS number(s): 47.55.dd, 47.55.dp, 66.25.+g, 05.70.Ln

\section{INTRODUCTION}

Although the behavior of equilibrium inhomogeneous fluid systems has been rationalized within the framework of the density functional theory, the description of out-of-equilibrium situations as for instance created by an external temperature gradient has been poorly explored [1]. This latter physical situation is relevant to experiment where a metallic nanoparticle surrounded by liquid water is heated up by a laser source, thus creating a very large local temperature gradient $\sim 1 \mathrm{~K} / \mathrm{nm}$ in the fluid. The possibility to generate such gradients open the way to plethora of applications. Among others, we can mention the design of self-propellers using colloids that move in the temperature gradient they create $[2,3]$, the possibility to measure heat currents flowing across a molecule [4] or on a more fundamental side the generalization of Einstein law for the diffusion of a Brownian particle [5,6].

Another unique situation may be offered when the laser heating is strong enough to drive liquid phase change in the vicinity of the hot nanoparticle. The so-called boiling under very large temperature gradient is commonly related to the crossing of the spinodal line, which occurs at a temperature just below the critical fluid temperature. After the crossing of the relevant transition line, a thin vapor layer may be allowed to form a "nanobubble" which further expands in the liquid. The production of nanobubbles has been recently evidenced experimentally, based on x-ray scattering [7] or direct optical measurements [8,9], and it has been confirmed by simulations $[10,12]$. Depending on the size of the bubbles produced, different applications have been developed so far. Gold nanoparticles exposed to short laser radiation allow for the generation of short-lived nanobubbles of vapor which can be used as a tool for diagnosis and treatment of tumors [13]. At the other extreme, continuous irradiation of nanoparticles by focused solar light leads to the production of steam at temperatures higher than the saturation temperature at ambient pressure, which can be used for sterilization and solar energy recovery among other interesting applications [8].
While the crossing of the liquid spinodal seems to be the criterion driving nanobubble generation $[7,10]$, the thermodynamics of the nanobubble once formed remain elusive. In fact, liquid phase change in a temperature gradient is a problem not amenable to simple analytical treatment. Here the description is still harder because of the strong curvature of the nanobubble. This amounts to the following simple questions: What is the temperature inside the bubble? The pressure? How can we describe the thermodynamic state of the vapor bubble during the growth and collapse? How does the nanobubble support very large Laplace pressures? The different physical mechanisms at play in the energy exchange between the hot nanoparticle and the bubble have been sketched in Ref. [11], showing the difficulty in building up a simple theoretical treatment.

In previous work [12] we analyzed theoretically the nanobubble dynamics using hydrodynamics phase field simulations. We focused on the nanobubble radius and showed that it can be well described by a "Rayleigh-Plesset" equation, classically used to analyze cavitation phenomena. This analysis allowed us to conclude that the growth of the nanobubble is best described by an adiabatic evolution, while the collapse is isothermal. This analysis was confirmed by a molecular dynamics (MD) study in Ref. [10], and the good behavior of the macroscopic Rayleigh-Plesset theory opens the question of a thermodynamic macroscopic-like description of the nanobubble. MD is a very valuable approach because it allows a molecular-scale description of the phenomena with simple assumptions on the molecular interactions. Unfortunately, due to the transient nature of the bubbles, and the very low density of the vapor phase, a quantitative analysis of the thermodynamic states inside the bubble is very difficult. Our thermodynamic approach is a good complement of MD in this case, since we directly have information on the thermodynamic states and the energy fluxes. Furthermore, the present analysis is not restricted to nanometerscale systems, which is a key point for any investigation of the propagation of the pressure wave induced by the formation of the bubble. In this article we focus on the temperature and the pressure in the nanobubble, 
after its formation. In particular, we show that the initial very large temperature gradient under which the nanobubble is formed quickly relaxes, as the energy flow from the nanoparticle switches from conductive to ballistic. This enables a "bulk"-like treatment of the vapor bubble thermodynamics. Subsequently the temperature inside the vapor is found to be homogeneous while any temperature gradient is localized in the liquid in the vicinity of the nanobubble. This is the result of the ballistic nature of the heat flux inside the vapor nanobubble, owing to its submicronic thickness. We also discuss the evolution of the pressure inside the vapor nanobubble. We show that a pressure wave is first emitted from the hot nanoparticle, and then viscous forces relax the internal vapor pressure, which reaches Laplace pressure when the nanobubble has stopped growing.

The article is structured as follows: We first depict the physical situation that we address theoretically in Sec. II. In Sec. III we describe the hydrodynamic phase field model used to probe the nanobubble dynamics. The relaxation of the temperature gradient during boiling is first discussed in Sec. IV, before concentrating on the internal pressure relaxation inside the vapor bubble in Sec. V.

\section{MOTIVATIONS: SKETCH OF THE SYSTEM STUDIED}

We briefly depict in this section the physical situation that we will address theoretically. A nanoparticle having a radius $R_{\mathrm{np}}>2 \mathrm{~nm}$ is surrounded by liquid water, and the whole system is initially at thermal equilibrium at room temperature. We break this equilibrium by heating up the particle during a very short time at a temperature $T_{p}$ through the interaction with a laser pulse of short duration. The article aims at describing the state of the fluid, subsequent to the heating of the particle as represented in Fig. 1. A quantity of prime importance at the nanoscale is the thermal boundary resistance between the particle and the fluid, the latter being defined from the thermal flux flowing across the solid-water interface and the temperature jump:

$$
R=\frac{T_{p}-T_{s}}{j},
$$

where $T_{p}$ is the nanoparticle temperature, $T_{s}$ is the fluid temperature at the nanoparticle surface, and $j$ is the heat flux density. For a gold-water interface, the interfacial conductance $1 / R$ is typically between 50 and $150 \mathrm{MW} / \mathrm{m}^{2} / \mathrm{K}$, depending on the presence of a self-assembled monolayer of surfactants between gold and water [14-17].

The three points that we aim at investigating in this article are the following: (1) How does the nanobubble grow in the steep temperature gradient? (2) What is the temperature inside the bubble? The pressure? (3) How does the nanobubble formed accomodate the very large Laplace pressures induced by its strong curvature?

\section{MODEL}

Modeling the nucleation and growth of transient vapor bubbles around GNPs is a formidable challenge for a theoretician, because of the highly out-of-equilibrium nature of the problem, the large capillary effects present at the nanoscale, and the occurrence of phase change under very large temperature gradients. In addition, energy transport inside the bubble is no longer diffusive but ballistic, because the dimensions of the nanobubbles are smaller than the vapor molecules' mean free path. Molecular dynamics simulations, although offering the flexibility to model the relevant situations, become prohibitive to simulate the dynamics of the nanobubbles, because of the long-range effects involved [10,18].

Alternatively, a hydrodynamic model based on a free energy density has been successfully applied to address interfacial heat transport and boiling at nanoscale [19,20]. We have extended basically this model to account for a finite thermal boundary resistance between the GNPs and the surrounding fluid, and also the possibility of ballistic energy transport inside the bubble. Since the model was already presented in Ref. [12], we shall not give all the details, but focus on the main physical ingredients concerning the fluid dynamics and the different energy fluxes.

\section{A. Fluid model}

We solve the hydrodynamic equations to describe the dynamics of the fluid around the nanoparticle:

$$
\begin{aligned}
\frac{\partial \rho}{\partial t}+\nabla \cdot(\rho \mathbf{v}) & =0 \\
m \rho\left(\frac{\partial \mathbf{v}}{\partial t}+\mathbf{v} \cdot \nabla \mathbf{v}\right) & =-\nabla \cdot(\mathbf{P}-\mathbf{D}), \\
m \rho c_{v}\left(\frac{\partial T}{\partial t}+\mathbf{v} \cdot \nabla T\right) & =-l \nabla \cdot \mathbf{v}+\nabla \cdot(\lambda \nabla T)+\mathbf{D}: \nabla v
\end{aligned}
$$

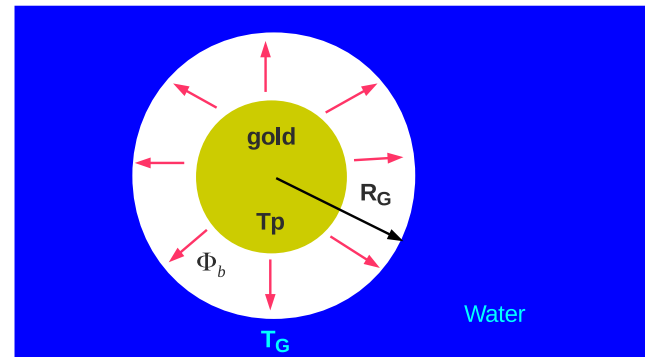

FIG. 1. (Color online) Sketch of the system considered: a gold nanoparticle in water, initially heated by a strong laser pulse of short duration, as represented by the green line in the left panel. The transient radial temperature profile across the particle is shown with blue lines in the left panel, emphasizing the effect of a finite thermal resistance at the interface. The GNP/laser interaction may result in the formation of a vapor nanobubble surrounding the GNP, as illustrated in the right panel. 
TABLE I. Thermophysical parameters in the liquid (top row) and in the vapor (bottom row) at $297 \mathrm{~K}$ in SI units unless specified.

\begin{tabular}{lccccc}
\hline \hline Density $^{\mathrm{a}}$ & $C_{v}{ }^{\mathrm{b}}$ & $\lambda$ & $\eta$ & $l$ & $\gamma$ \\
\hline $997 \times 10$ & 4.13 & 0.606 & $8.98 \times 10^{-4}$ & $5.4 \times 10^{8}$ & $72.0 \times 10^{-3}$ \\
$2.22 \times 10^{-2}$ & 1.44 & 0.019 & $9.9 \times 10^{-6}$ & 6881 & \\
\hline \hline
\end{tabular}

${ }^{\mathrm{a}} \mathrm{kg} / \mathrm{m}^{3}$

${ }^{\mathrm{b}} \mathrm{kJ} / \mathrm{kgK}$.

where $\rho, \mathbf{v}, T$ stand, respectively, for the number density, the velocity field, and the temperature field; $m$ is the mass of a fluid molecule. $c_{v}, l, \lambda$, are the fluid specific heat, Clapeyron coefficient $l=T\left(\frac{\partial P}{\partial T}\right)_{\rho}$, and thermal conductivity. $\mathbf{D}$ and $\mathbf{P}$ stand for the dissipative stress tensor and pressure tensor. The symbol ":" represents a dyadic product.

The dissipative stress tensor writes

$$
D_{\alpha \beta}=\eta\left(\partial_{\alpha} v_{\beta}+\partial_{\beta} v_{\alpha}-\frac{2}{3} \nabla \cdot \mathbf{v} \delta_{\alpha \beta}\right)+\mu \nabla \cdot \mathbf{v} \delta_{\alpha \beta} .
$$

The bulk viscosity $\mu$ is related to the shear viscosity $\eta$ through $\mu \simeq 5 \eta / 3$, a reasonable approximation for hard spheres liquids [21]. The thermophysical and transport coefficients of liquid water and vapor at $297 \mathrm{~K}$ and atmospheric pressure are summarized in Table I, together with the water surface tension $\gamma$. Since the density $\rho$ in (2) is a field with large spatial variations, we need to account for the variation of the thermophysical and transport coefficients with the local density. We choose a linear relationship between those parameters and the density. As an example, the local shear viscosity is given by

$$
\eta(r)=\eta_{\text {vap }}+\frac{\rho(r)-\rho_{\text {vap }}}{\rho_{\text {liq }}-\rho_{\text {vap }}}\left(\eta_{\text {liq }}-\eta_{\text {vap }}\right),
$$

where the subscripts vap and liq refer to the bulk values from Table I at $297 \mathrm{~K}$.

The pressure tensor and the Clapeyron coefficient are related to the local thermodynamic pressure, and the density gradient according to

$$
\begin{aligned}
P_{\alpha \beta} & =\left[P_{\mathrm{VdW}}-w \rho \Delta \rho+\frac{w}{2}(\nabla \rho)^{2}\right] \delta_{\alpha \beta}+w \partial_{\alpha} \rho \partial_{\beta} \rho, \\
l & =T\left(\frac{\partial P_{\mathrm{VdW}}}{\partial T}\right)_{\rho} .
\end{aligned}
$$

These latter expressions are dependent on the local state of the fluid, through a free energy density $f$ consisting of a bulk van der Waals free energy density $f_{\mathrm{VdW}}$ and a capillary term. This free energy density $f_{\mathrm{VdW}}$ and the corresponding thermodynamic pressure write

$$
\begin{gathered}
f=f_{\mathrm{VdW}}+\frac{w}{2}|\nabla \rho|^{2}, \\
f_{\mathrm{VdW}}=\rho k_{B} T\left[\ln \left(\frac{\rho \Lambda^{3}}{1-\rho b}\right)-1\right]-a \rho^{2}, \\
P_{\mathrm{VdW}}=\frac{\rho k_{B} T}{1-\rho b}-a \rho^{2} .
\end{gathered}
$$

The parameters $a, b$, and the De Broglie wavelength $\Lambda$ in Eqs. (7) and (8) are set so as to represent the density of liquid water at $297 \mathrm{~K}$ and atmospheric pressure and its critical parameters. The relation between the parameters in Eq. (7) and the critical parameters are given by

$$
\begin{aligned}
& P_{c}=\frac{a}{27 b^{2}}=22 \mathrm{MPa}, \\
& \rho_{c}=\frac{1}{3 b}=322 \mathrm{~kg} / \mathrm{m}^{3}, \\
& T_{c}=\frac{8 a}{27 b k_{B}}=647.3 \mathrm{~K} .
\end{aligned}
$$

The parameter $w$ appearing in the square gradient term in the pressure tensor $\mathbf{P}$ quantifies the strength of the surface tension forces in the fluid since it prevents the fluid from developing steep gradients. Its magnitude was set so as to match the surface tension $\gamma$ of water at $T=297 \mathrm{~K}$ :

$$
\gamma=\sqrt{2 w} \int_{\rho_{\mathrm{vap}}}^{\rho_{\mathrm{liq}}} \sqrt{f_{\mathrm{VdW}}(\rho)-\mu_{\mathrm{eq}}(T) \rho+P_{\mathrm{eq}}(T)} d \rho,
$$

where $\rho_{\text {vap }}$ and $\rho_{\text {liq }}$ are the coexistence vapor and liquid densities on each side of a liquid-vapor interface at temperature $T$. $\mu_{\mathrm{eq}}(T)$ and $P_{\mathrm{eq}}(T)$ are the chemical potential and pressure of both the liquid and vapor bulk phases at equilibrium at temperature $T$, as given by the van der Waals equation of state.

The treatment of the Clapeyron coefficient $l$ was done separately, and for this coefficient we considered a dependence in temperature in addition to the dependence in density. Indeed, unlike the other parameters, $l$ displays important variations with the temperature:

$$
l(\rho, T)=l_{\text {vap }}(T)+\frac{\rho(r)-\rho_{\text {vap }}}{\rho_{\text {liq }}-\rho_{\text {vap }}}\left[l_{\text {liq }}(T)-l_{\text {vap }}(T)\right],
$$

where $l_{\text {liq }}(T)$ and $l_{\text {vap }}(T)$ are the temperature-dependent values of the Clapeyron coefficient at a given temperature $T$, as depicted in Fig. 2. The values of $l_{\text {vap }}(T)$ and $l_{\text {liq }}(T)$ have been extracted from the experimental data as provided by the

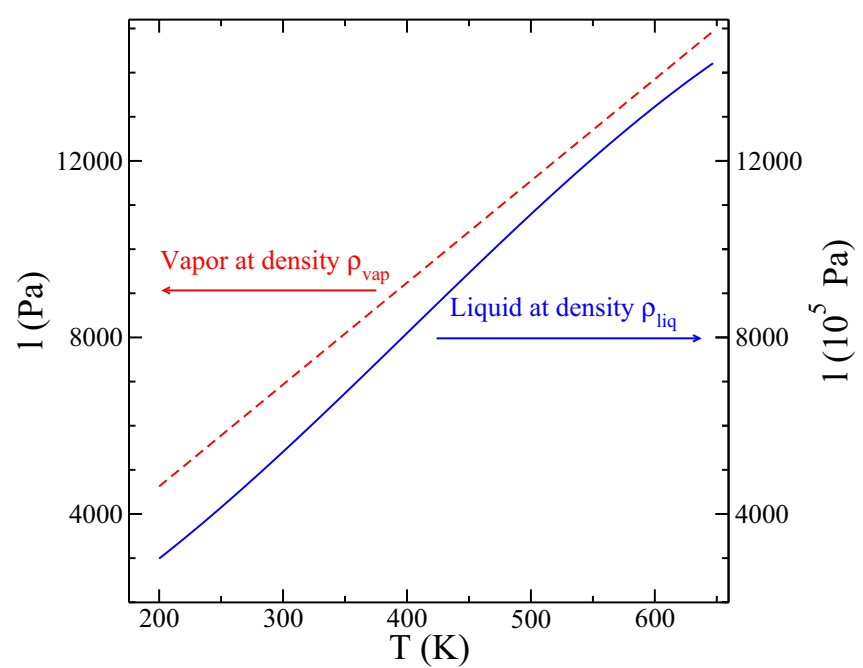

FIG. 2. (Color online) Evolution of the Clapeyron coefficient with temperature in a liquid of density $\rho_{\text {liq }}$ (blue solid line) and in a vapor of density $\rho_{\text {vap }}$ (red dashed line). The coefficients have been obtained from experimental data [22]. 
NIST [22]. The reference $\rho_{\text {liq }}$ and $\rho_{\text {vap }}$ have been taken to be the saturation density at room temperature.

\section{B. Nanoparticle}

We now discuss the interaction of the fluid with the nanoparticle. We account for the wettability of the fluid, the interface resistance for thermal conduction, the continuity of pressure, and the no-slip conditions. This gives the following boundary conditions at the fluid-GNP interface:

$$
\begin{gathered}
(\nabla \rho)_{\mathrm{r}=\mathrm{R}_{\mathrm{np}}}=\frac{\Phi}{w}, \\
(\nabla T)_{\mathrm{r}=\mathrm{R}_{\mathrm{np}}}=-\frac{G}{\lambda}\left(T_{\mathrm{np}}-T_{s}\right), \\
\mathbf{v}\left(r=R_{\mathrm{np}}\right)=\mathbf{0}, \\
{\left[\nabla \cdot \mathbf{P}\left(r=R_{\mathrm{np}}\right)\right] \cdot \mathbf{n}=0 .}
\end{gathered}
$$

The potential $\Phi$ in (12) quantifies the wetting of the fluid $[19,23]$. It can be directly related to the contact angle of the fluid with a given solid, as expressed by Eq. (16), and illustrated in Fig. 3. In all the following, we choose the value for $\Phi$ corresponding to a contact angle of $50^{\circ}$ at $297 \mathrm{~K}$ :

$\cos \theta=1-\frac{1}{\gamma} \int_{\rho_{v}^{s}}^{\rho_{l}^{s}}\left[\sqrt{2 w\left(f_{\mathrm{VdW}}(\rho)-\mu_{\mathrm{coex}} \rho+P_{\mathrm{coex}}\right)}+\phi\right] d \rho$.

The interface conductance $G$ in (13) will be discussed later in the description of the nanoparticle temperature. Equation (15) stands for the continuity of pressure at the particle surface, $\mathbf{n}$ being the unit vector perpendicular to the GNP surface pointing outwards.

For the metal nanoparticle, the temperature $T_{\mathrm{np}}$ is assumed to be uniform, a reasonable hypothesis owing to the large conductivity of the metal. This assumption was confirmed in a simulation work for quasi-instantaneous heating of the nanoparticle, and for continuous illumination in the limit of

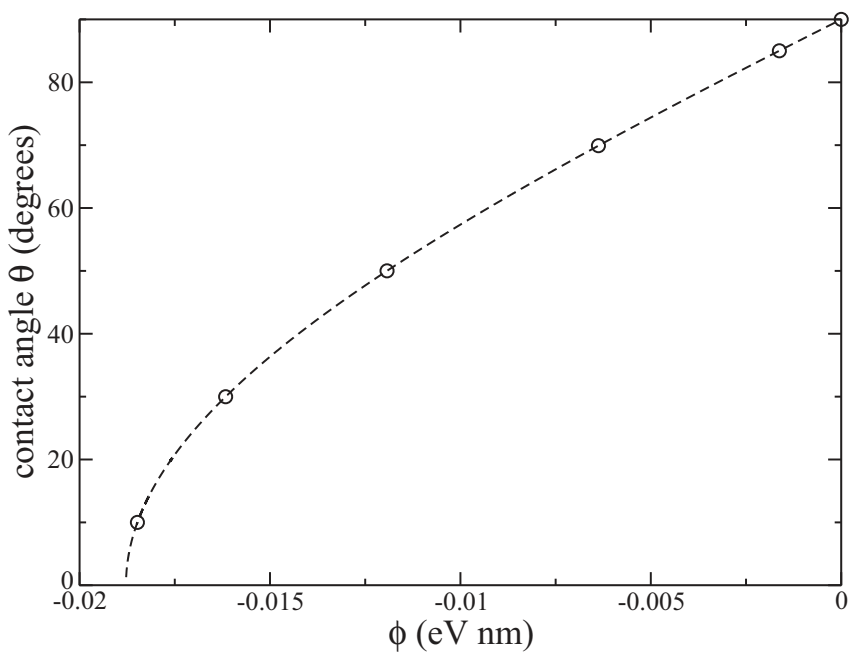

FIG. 3. Equilibrium contact angle as a function of the solid-wall parameter $\Phi$ appearing in Eq. (12). small GNP radii $R_{\mathrm{np}}<50 \mathrm{~nm}$, by Ref. [24]. The temporal evolution of the nanoparticle temperature is described by

$$
\begin{gathered}
V_{\mathrm{np}} C_{\mathrm{np}} \frac{d T_{\mathrm{np}}}{d t}=F \sigma_{\mathrm{np}} \frac{\Pi\left(t / t_{p}\right)}{t_{p}}-S_{\mathrm{np}} \phi, \\
\phi=\phi_{c}+\phi_{b}, \quad \phi_{c}=G\left(T_{\mathrm{np}}-T_{s}\right), \\
\phi_{b}=\alpha \rho_{s} \sqrt{\frac{2 k_{B}^{3}}{m}}\left(T_{\mathrm{np}}^{3 / 2}-T_{G}^{3 / 2}\right),
\end{gathered}
$$

where $S_{\mathrm{np}}, V_{\mathrm{np}}$ are the nanoparticle surface and volume, respectively, and $C_{\mathrm{np}}=2500 \mathrm{~kJ} / \mathrm{m}^{3} \mathrm{~K}$ is the gold specific heat. The laser interaction is described by the size-dependent GNP absorption cross section $\sigma_{\text {np }}$ as given in Ref. [7], the fluence of the laser pulse $F$, and the gate function $\Pi\left(t / t_{p}\right)=1$ if $0<t<t_{p}$ and 0 otherwise, where $t_{p}$ stands for the duration of the GNP heating.

The parameter $t_{p}=7 \mathrm{ps}$ used here is larger than the pulse duration. Since we consider femtosecond pulses, the relevant time for our study is the electron-phonon coupling time as described by Ref. [25], which determines the GNP phonon equilibration time: for $t \geqslant t_{p}$, the GNP lattice has received all the energy initially provided to its electron gas by the laser pulse. The electron interaction with the pulse prior to that coupling is not accounted for, as its characteristic time is much smaller than $t_{p}$. Other phenomena such as particle melting are not modeled in (17), but we discussed their importance in Ref. [12].

The last term in (17) describes the energy flux flowing away from the nanoparticle. It is decomposed here, in a conductive term $\phi_{c}$ and a ballistic heat flux $\phi_{b}$ which is nonvanishing when the fluid locally vaporizes. The conductive flux is $\phi=$ $\phi_{c}=G\left(T_{\mathrm{np}}-T_{s}\right)[14,18,26-28]$ where the thermal boundary conductance $G$ characterizing the gold-fluid interface writes $G=G_{0}[1+\cos (\theta)]$ and depends on the contact angle $\theta$ $[15,16]$. The constant $G_{0}$ has been taken to depend on the fluid surface density $\rho_{s}$, so as to account for the drop of the conductance when the fluid locally vaporizes. We have considered $G_{0}=\frac{1}{2}\left\{\left(G_{\mathrm{L}}-G_{\mathrm{V}}\right) \tanh \left[\left(\rho_{s}-\rho_{c}\right) / \delta \rho\right]+G_{\mathrm{L}}+G_{\mathrm{V}}\right\}$, where $G_{\mathrm{L}}$ and $G_{\mathrm{v}}$ denote the conductance when the fluid is in the liquid state and vapor state. respectively, $\delta \rho=0.025 / b$ allows us to describe the sharp transition between the two limiting behaviors: liquid or vapor. The value of $G_{\mathrm{L}}=90 \mathrm{MW} \mathrm{m} \mathrm{K}^{-1}$ has been extracted from available experimental and simulation results for a gold-water interface. As for the value of $G_{\mathrm{V}}$, we have assumed $G_{\mathrm{V}}=\frac{3}{2} \rho_{v} \sqrt{\frac{2 k_{B}^{3}}{m}} T_{\infty}$, a form which is inspired by the kinetic theory of gas, and which yields a value in agreement with available experimental and simulation data [29]. Importantly, this value is typically 20 times lower than $G_{\mathrm{L}}$.

The ballistic heat flux $\phi_{b}$ takes nonnegligible values when the fluid locally vaporizes. The conductive heat flux from the nanoparticle to the fluid is small, and the GNP energy is transferred to the fluid through ballistic transport in the vapor nanobubble. This energy flux depends on the temperature of the particle and that of the fluid at the position of the liquid-vapor interface. The expression of the ballistic flux is given in Eq. (19), where $\alpha$ is a dimensionless accommodation coefficient here set to $0.1[29,30], \rho_{s}$ is the fluid density at the particle surface, and $T_{G}$ is the temperature of the fluid at the 
Gibbs position of the liquid-vapor interface. The expression of the ballistic flux in (19) is inspired by the theory of energy transport in a Knudsen layer [30]. This ballistic transport is relevant only for bubbles thickness $e_{\mathrm{b}}$ small enough so that the vapor can be considered as a Knudsen gas. This criterion is obeyed for Knudsen number $K n=\lambda_{\mathrm{mfp}} / e_{\mathrm{b}}<1$, where $\lambda_{\mathrm{mfp}} \simeq 100 \mathrm{~nm} \mathrm{[31]}$ is the mean free path of water molecules inside the bubble.

We choose to define the radius of the bubble $R_{b}$ as the location of the Gibbs surface where the average density lies: $\rho\left(R_{b}\right)=\left(\rho_{\min }+\rho_{\max }\right) / 2 . \rho_{\min }$ and $\rho_{\max }$ stand, respectively, for the minimum and maximum densities in the fluid. It should be finally mentioned that when a bubble appears, energy conservation is ensured through the addition of a flux $\phi_{b}^{\prime}=\phi_{b} R_{\mathrm{np}}^{2} / R_{b}^{2}$ localized at the liquid-vapor interface, and which represents the amount of energy per unit of time that is received by the liquid stemming from the ballistic flux $\phi_{b}$.

\section{Boundary conditions}

In our simulations we implemented damping conditions at the boundaries to avoid any reflection of the pressure wave that may induce a premature collapse of the bubble. The method we use is inspired by the perfectly match layers (PML) $[32,33]$ used in finite element methods. To do so, we artificially damped the gradient of any field so that the boundaries do not generate reflected waves that may perturb the dynamics in the simulation cell. As an example, for the density gradient, we used

$$
\nabla \rho(r)=\frac{1}{1+\epsilon(r)} \frac{\partial \rho}{\partial r},
$$

where $\epsilon(r)$ is a function linearly increasing with the radius if $r>r_{\mathrm{PML}}$ and $\epsilon(r)=0$ otherwise. As will be discussed later, the generation of nanobubbles implies the emission of a pressure wave to relax the excess pressure at the nanoparticle surface. This pressure wave flows away from the particle, and we need to ensure that the system boundaries will not artificially reflect them toward the GNP. After several tests, we chose a slope 0.1 for the evolution of $\epsilon(r)$ versus radius so $\epsilon(r)=0.1\left(r-r_{\mathrm{PML}}\right)$. A smaller value of this slope leads to waves flowing through the damping region and creates a depletion zone after the passage of the wave. A higher value blocks the pressure wave and locally creates an area of excess pressure at the PMLs that act as a solid wall and reflects the wave towards the nanoparticle.

We study particles of radii varying from 2 to $50 \mathrm{~nm}$. We consider a spherical symmetry that allows us to focus only on the radial component of the fields. All the spatial derivatives appearing in the conservation equations (2) and the boundary conditions (12)-(15) are calculated using spherical geometry.

The size of the simulation cell, centered at the position of the GNP center, is $L=260 \mathrm{~nm}$ for $R_{\mathrm{np}}<50 \mathrm{~nm}$ and $L=390 \mathrm{~nm}$ for $R_{\mathrm{np}}=50 \mathrm{~nm}$. The size of the damping area $L-R_{\mathrm{np}}-r_{\mathrm{PML}}$ is $65 \mathrm{~nm}$. With these values, the region where the fluid dynamics is undamped has a spatial extension $L-R_{\mathrm{np}}$, which is more than $140 \mathrm{~nm}$ large. We use a time step of $0.2 \mathrm{fs}$ and a lattice step of $0.07 \mathrm{~nm}$. The velocity field is calculated on a staggered grid, shifted from the main grid by half a lattice step.

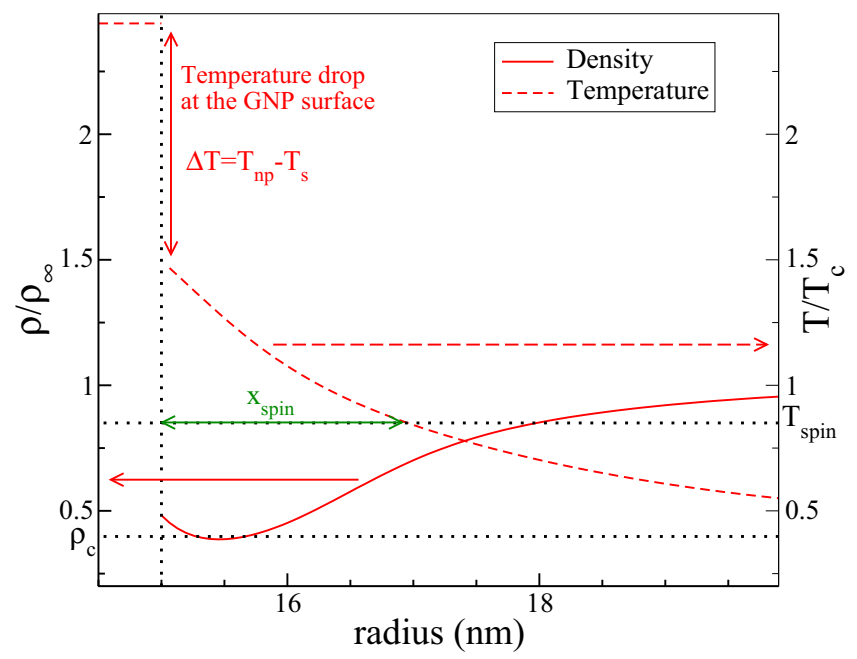

FIG. 4. (Color online) Density and temperature profiles in the fluid surrounding a nanoparticle when a bubble forms. Here the particle radius is $R_{\mathrm{np}}=15 \mathrm{~nm}$ and the laser fluence $F=101.25 \mathrm{~J} / \mathrm{m}^{2}$. The dotted lines show, from top to bottom, the position of the spinodal temperature $T_{\text {spin }}$, the average density, and the critical density $\rho_{c}$. The red dashed arrow on the left emphasizes the temperature drop at the surface of the nanoparticle. On this figure we indicate the instantaneous position of the spinodal crossing, at a distance $x_{\text {spin }}$ from the GNP surface. This corresponds to the crossing of the spinodal line, at the temperature $T_{\text {spin }}$.

\section{THERMODYNAMIC ANALYSIS}

In this section we aim at providing a thermodynamic description of the vapor nanobubble, after its formation. In all the following, we consider that a bubble is formed if there is at least one point in the fluid where the density is below the water critical density $\rho_{c}=322 \mathrm{~kg} / \mathrm{m}^{3}$. We

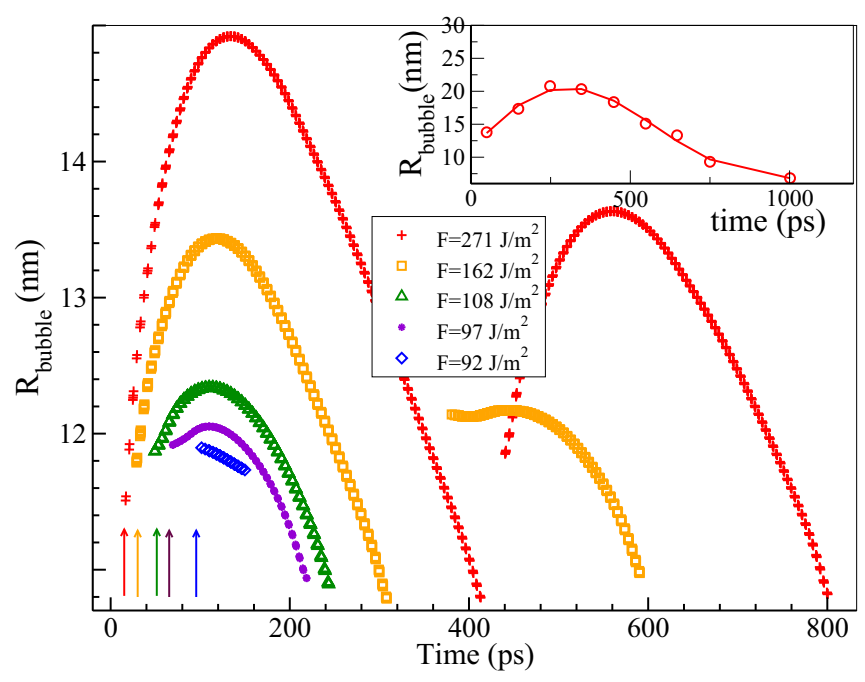

FIG. 5. (Color online) Temporal evolution of the nanobubble radius $R_{b}$ around a $10 \mathrm{~nm}$ GNP for different pulse fluences (the higher fluence giving the larger bubbles). A secondary bubble is observed for strong pulses. The arrows indicate the appearance time for each simulation. The inset displays the nanobubble radius observed experimentally around a $9 \mathrm{~nm}$ diameter nanoparticle [7]. The solid line is a guide to the eye. 

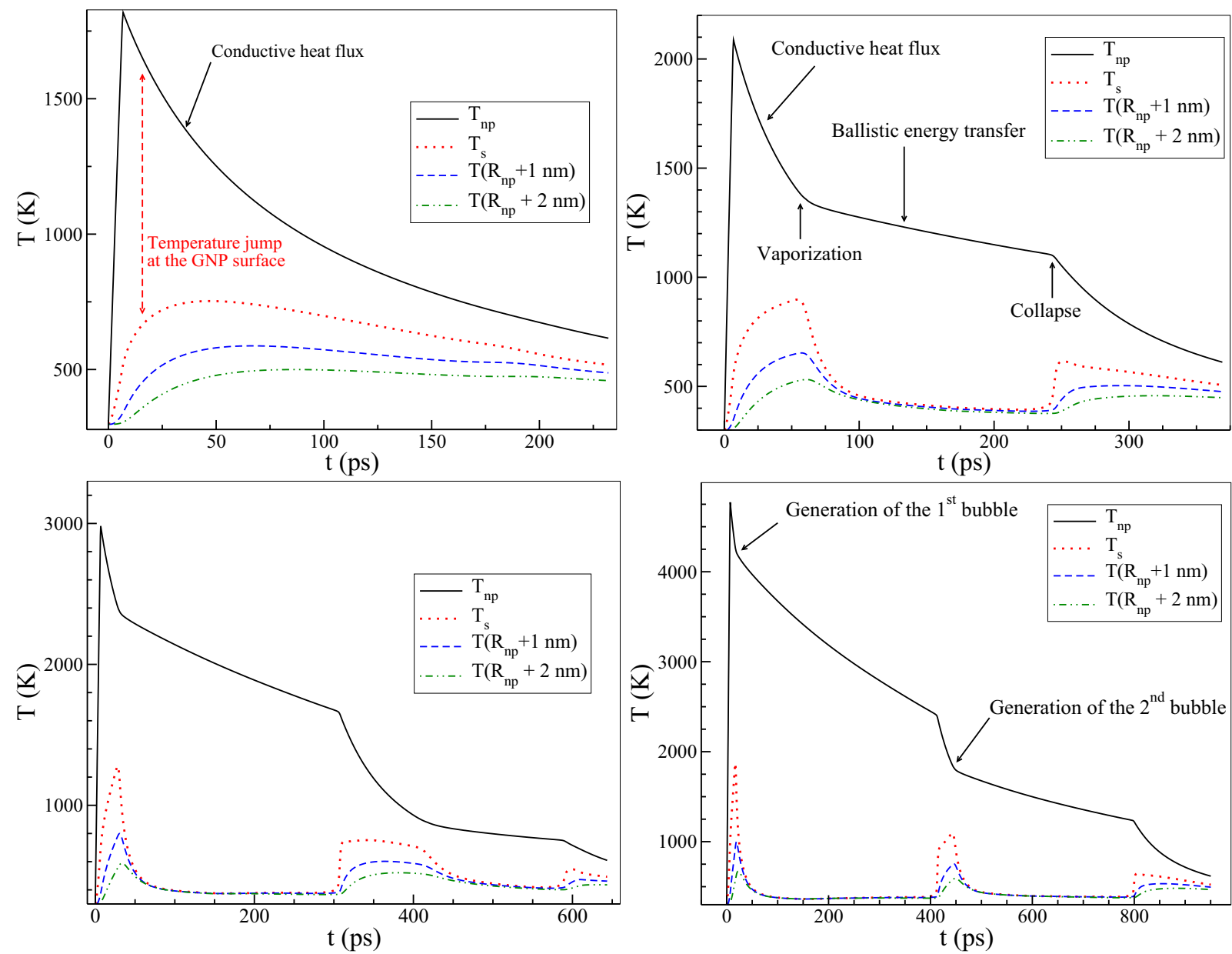

FIG. 6. (Color online) Temperature of the particle $\left(T_{\mathrm{np}}\right)$ and the fluid at the surface $\left(T_{s}\right), 1 \mathrm{~nm}$ from the surface, and $2 \mathrm{~nm}$ from the surface as a function of time. Here $R_{\mathrm{np}}=10 \mathrm{~nm}, F=92 \mathrm{~J} / \mathrm{m}^{2}$ (top left), $F=108 \mathrm{~J} / \mathrm{m}^{2}$ (top right), $F=162 \mathrm{~J} / \mathrm{m}^{2}$ (bottom left), and $F=271 \mathrm{~J} / \mathrm{m}^{2}$ (bottom right).

already showed [12] that nanobubble formation coincides with spinodal crossing in the fluid, at a distance $x_{\text {spin }}$ between 1 and $2 \mathrm{~nm}$ from the GNP surface, in liquid water. This occurs at a temperature $T_{\text {spin }} \simeq 0.9 T_{\mathrm{c}} \simeq 550 \mathrm{~K}$ for liquid water. Spinodal crossing corresponds to a fluence-energy per unit of GNP cross section, larger than a size-dependent threshold, and we assume that we are working under these condition. Here we are mostly interested in the dynamics of the nanobubble after the vaporization process.

Figure 4 displays an example of density profile, at the moment when a bubble forms. In this figure, the gradient of density at the particle surface is due to the wettability of the fluid as described by (12). The red dashed arrow on the left emphasizes the temperature drop at the surface of the nanoparticle due to the interfacial thermal resistance that delays the energy transfer from the GNP to the fluid.

Figure 5 displays the temporal evolution of the bubble radius, for different fluences beyond the threshold. This figure is quite similar to Fig. 2 in our previous article [12]. The radius of the nanobubble is determined based on the instantaneous location of the Gibbs dividing surface.

For fluences just above the threshold $\left(F=92 \mathrm{~J} / \mathrm{m}^{2}\right.$ in Fig. 5), we do observe a short-lived thin vapor layer. On increasing the laser fluence, mature bubbles are generated which first expand in the liquid and reach a maximal size before collapsing. A second bubble may be produced, if the fluence is high enough $\left(F \geqslant 162 \mathrm{~J} / \mathrm{m}^{2}\right.$ in Fig. 5). The production of this second nanobubble is explained by the compression of the vapor bubble at the ultimate stage of the collapse, leading to nanoparticle heating. For strong pulses the dynamics of the nanobubble is found to be asymmetrical, the growth being faster than the collapse. This asymmetry is also observed experimentally (and numerically in Ref. [10]) as evidenced by the inset of Fig. 5, and it was explained in Ref. [12] by the adiabatic nature of the bubble expansion, followed by the isothermal bubble collapse. We will revisit this scenario in this article on the basis of the thermodynamic analysis.

We analyze now the temperature of the nanoparticle before and after boiling, as represented in Fig. 6. On these figures, we have also displayed the temperature of the fluid at different distances from the GNP. We choose to focus on distances $1 \mathrm{~nm}$ and $2 \mathrm{~nm}$ from the nanoparticle, which for a $10 \mathrm{~nm}$ GNP corresponds to the typical value of the characteristic distance $x_{\text {spin }}$ where the spinodal line is locally crossed. Figure 6 shows that the nanoparticle is rapidly heated by the pulse, while due to the interface resistance there is a delay in the heat transfer to the fluid. This results in an important temperature jump at the 

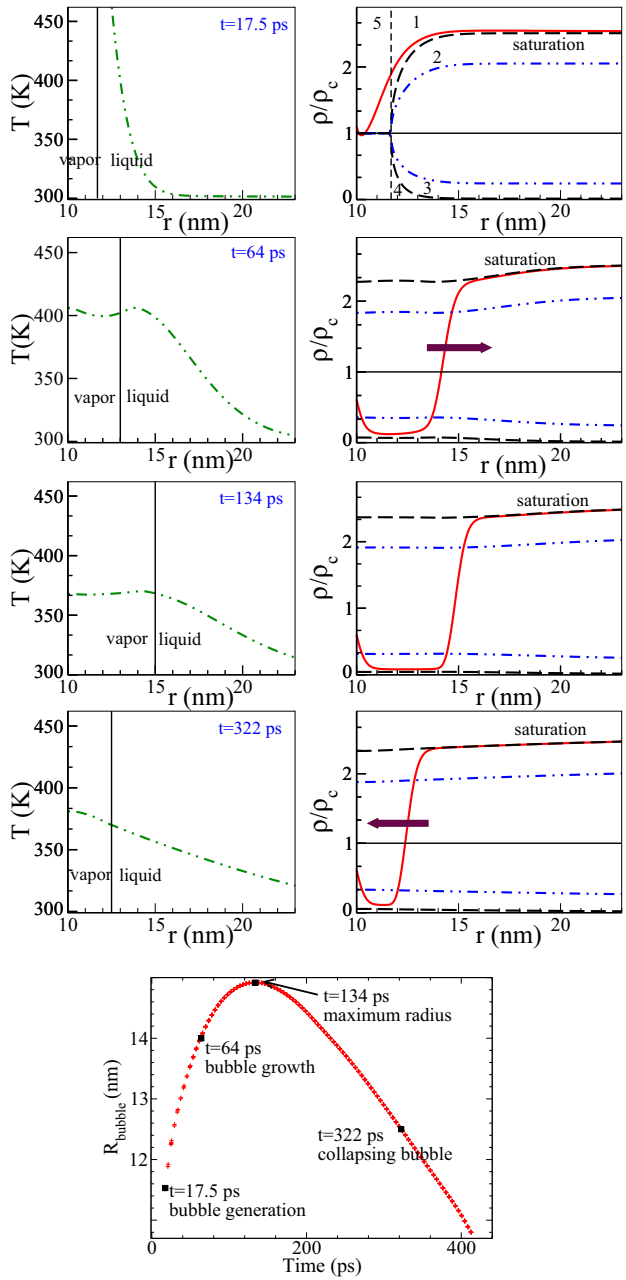

FIG. 7. (Color online) Radial temperature $\Delta T / T_{c}=\left(T-T_{\infty}\right) / T_{c}$ (left panels) and density profiles $\rho / \rho_{c}$ (right panels), taken at different times during the growth or collapse of the bubble. Here $R_{\mathrm{np}}=10 \mathrm{~nm}$ and $\mathrm{F}=271 \mathrm{~J} / \mathrm{m}^{2}$. From top to bottom, the corresponding times are $t=17.5 \mathrm{ps}$ (bubble formation), $t=64 \mathrm{ps}$ (growth), $t=134$ ps (maximal radius), and $t=322 \mathrm{ps}$ (collapse). The corresponding evolution of the radius is given in the bottom panel, where the black squares indicate the different times when the temperature and density profiles have been calculated. On the temperature profiles: the vertical solid lines show the instantaneous position of the liquid-vapor interface. On the density profiles: the black dashed lines show the local temperature-dependent values of the saturation limits. The dashed-dotted black lines show the local temperature-dependent values of the spinodal limits. On the top of the figure we also identified the thermodynamic domains delimited by these curves: on the left, close to the GNP, the temperature of the fluid is higher than the critical temperature and the fluid is supercritical (5). For $r \geqslant 1.17 \mathrm{~nm}$ the temperature in water is below $T_{c}$, and the saturation and spinodal curves delimit the stable liquid region (1) on the top, the metastable liquid region (2), the unstable region at the center, and the metastable (3) and stable (4) vapor regions.

GNP surface, represented by a dashed arrow on the left figure of Fig. 6.

Before vaporization, we observe a temperature decrease that can be described by an exponential evolution $T_{\mathrm{np}}-T_{\infty} \sim$ $e^{-t / \tau}$ where $\tau=R C_{\mathrm{np}} / 3 G=60 \mathrm{ps}$ for a $10 \mathrm{~nm}$ particle. In this regime, the nanoparticle cooling is controlled entirely by the interface thermal conductance $G$. Note that for the lowest fluence analyzed, this exponential regime persists all over the time window, as only a short-lived thin vapor layer is produced and the density at the nanoparticle surface stays above the critical value due to the wettability of the fluid; see the top left panel of Fig. 6.

When the fluence is larger, and after vaporization occurs the nanoparticle cools down very slowly, as seen in Fig. 6. In this regime the energy transfer between the nanoparticle and the fluid switches from conductive to ballistic, and the nanoparticle cooling is controlled by the ballistic flux $\phi_{b}$ of Eq. (19). The transition between conductive and ballistic is accompanied by an enhanced temperature drop. This drop is explained by the large increase of the interface thermal resistance when vaporization occurs. As a result, the fluid in the vicinity of the nanoparticle cools down sharply and sees its temperature decreasing by hundredths of kelvins in a few picoseconds. After this drop, the temperature and density in the fluid close to the particle reach a steady value during more than 200 ps, as visible in the bottom panels of Fig. 6. Also importantly, the temperature in the vapor seems to become rapidly homogeneous, and the temperature gradient initially present before vaporization has relaxed significantly. The temperature gradient relaxation is best visualized in Fig. 7, which present the simultaneous evolutions of the temperature and density profiles across the vapor bubble and the liquid.

When a nanobubble forms, the temperature gradient is first very large on the order of several kelvins per angstroms as evidenced in the top panels of Fig. 7. At this moment, there is a region of limited spatial extension where the density is below $\rho_{c}$ and the liquid-vapor interface is diffuse. Just after the nanobubble generation, the temperature gradient has relaxed and the liquid-vapor interface becomes sharp. The relaxation of the temperature gradient is clearly seen in Fig. 8 for different laser fluences. Here we plotted the maximal temperature gradient in the fluid. The corresponding position

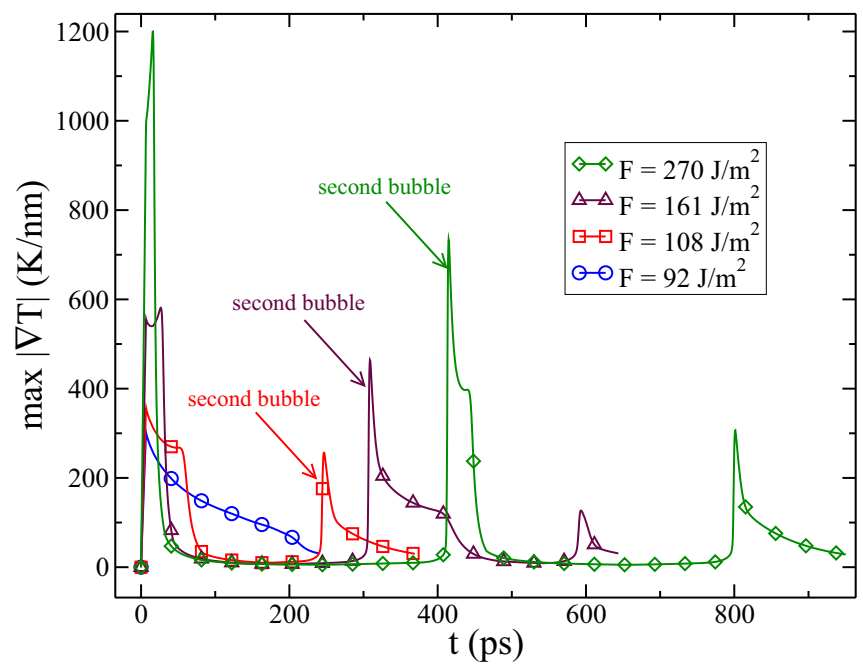

FIG. 8. (Color online) Relaxation of the maximal temperature gradient in the fluid for the different fluences considered in Fig. 6. Here $R_{\mathrm{np}}=10 \mathrm{~nm}$. 


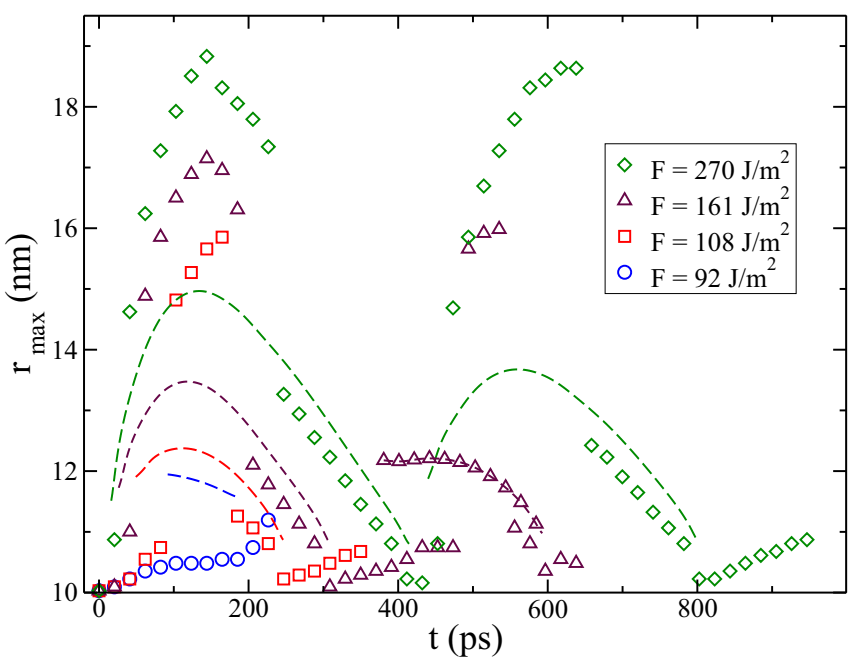

FIG. 9. (Color online) Position where the maximal temperature gradient occurs; see Fig. 8. The dashed lines show the instantaneous position of the nanobubbles for the different fluences analyzed.

where the maximum occurs is shown in Fig. 9. For the three highest fluences, the maximal temperature gradient is shown to relax quickly in a few picoseconds. This fast relaxation is explained by the huge drop of the thermal resistance from $1 / G_{\mathrm{L}}$ to $1 / G_{\mathrm{v}}$, when the bubble appears. This relaxation is accompanied by rapid cooling of the vapor in the bubble as testified by Fig. 7. Note that the temperature in the nanobubble is almost homogeneous. Some spatial variations may be seen during the early growth, which are certainly attributed to sound waves propagating in the vapor. The uniformity of the temperature in the vapor is due to the drop of the thermal boundary conductance from $G_{\mathrm{L}}$ to $G_{\mathrm{V}}$ subsequent to the switch from conductive to ballistic heat transfer in the vicinity of the nanoparticle. This fast drop yields an almost vanishing temperature gradient in the vapor, according to the boundary condition [Eq. (13)]. On the other hand, because the liquid collects the ballistic heat flux $\phi_{b}$ the local temperature gradient in the liquid $\frac{\partial T}{\partial r} \simeq-\phi_{b}^{\prime} / \lambda_{\text {liq }}$ may take appreciable values as shown in Fig. 7.

The localization of the temperature gradient is clearly confirmed by Fig. 9, which shows that the position where the maximal gradient occurs remains in the vicinity of the nanobubble interface. From a thermodynamic point of view, Fig. 7 shows that the liquid in the vicinity of the nanobubble follows the saturation line. Hence, we can conclude that just after the temperature relaxation step, the thermodynamic state of the liquid corresponds to saturation conditions. Also, importantly, we conclude from the inspection of the profiles that beyond a distance $x \simeq 2 \mathrm{~nm}$ from the GNP surface the density and temperature in the vapor are almost homogeneous. Hence, determining the thermodynamic path followed by the fluid at a fixed distance $x \simeq 2 \mathrm{~nm}$ from the GNP surface should provide information regarding the thermodynamic evolution of the vapor nanobubble.

We have performed such an analysis in Fig. 10, which represents the local thermodynamic state of the fluid at a given time, in a temperature-density diagram.

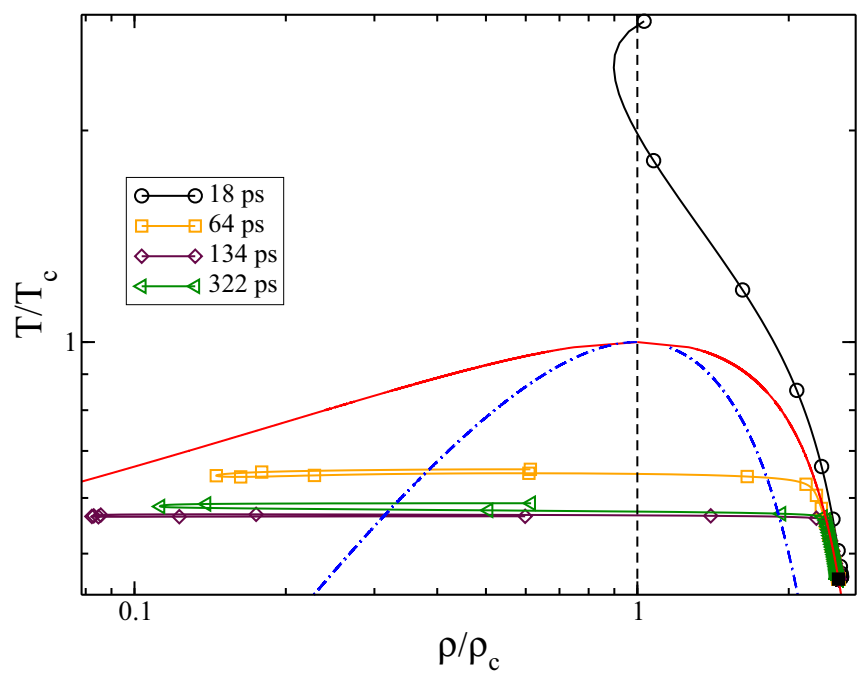

FIG. 10. (Color online) Local thermodynamic state of the fluid on a $T$ - $\rho$ diagram, taken at different times. The corresponding times are the same as Fig. 7: $t=17.5 \mathrm{ps}$ (bubble formation), $t=64 \mathrm{ps}$ (growth), $t=134 \mathrm{ps}$ (maximal radius), and $t=322 \mathrm{ps}$ (collapse). The fluence is here $\mathrm{F}=271 \mathrm{~J} / \mathrm{m}^{2}$ and $R_{\mathrm{np}}=10 \mathrm{~nm}$. The solid red line stands for the saturation curve, which delimits the stable and metastable phases for the vapor (on the left) and for the liquid (on the right). The dashed-dotted blue line stands for the spinodal curve, which represents the limit between the metastable phases and the unstable liquid-vapor zone (at the center).

The generation of the nanobubble leads to an important elevation of the temperature and pressure close to the particle, which generates a local supercritical region of small spatial extension. This sudden pressure increase induces a pressure wave, as we will analyze in Sec. V. This wave moves into the liquid and propagates quickly towards the boundaries of the system and does not interfere with the thermodynamics of the vapor nanobubble, which we discuss now.

Figure 10 confirms that the temperature inside the vapor nanobubble is almost homogeneous. Also, it is clear that the temperature gradient is localized in the liquid whose thermodynamic state follows the saturation line. The relative homogeneity of the temperature inside the bubble allows us to probe the thermodynamics of the vapor during the growth and collapse. To this end, we have plotted the evolution of the thermodynamic state of the fluid after the bubble formation in Fig. 11. We focused on two distances, at contact and at a distance $x=2 \mathrm{~nm}$ from the particle surface.

Even for the fluences considered, which are far from the generation threshold, the density at the GNP surface stays at best in the unstable region. The global behavior that is highlighted by Fig. 11 is the following: the liquid at $2 \mathrm{~nm}$ from the GNP surface is first heated and follows the saturation line. Then cooling proceeds and is accompanied by local vaporization and subsequent dilation of the vapor. Cooling stops when the nanobubble reaches its maximal radius, and the further thermodynamic evolution of the vapor is clearly isothermal, as seen in Fig. 11. It is immediately visible on Fig. 11 that the temperature during the collapse is constant and slightly above $T_{\infty}$. Any temperature gradient is then localized in the liquid. 

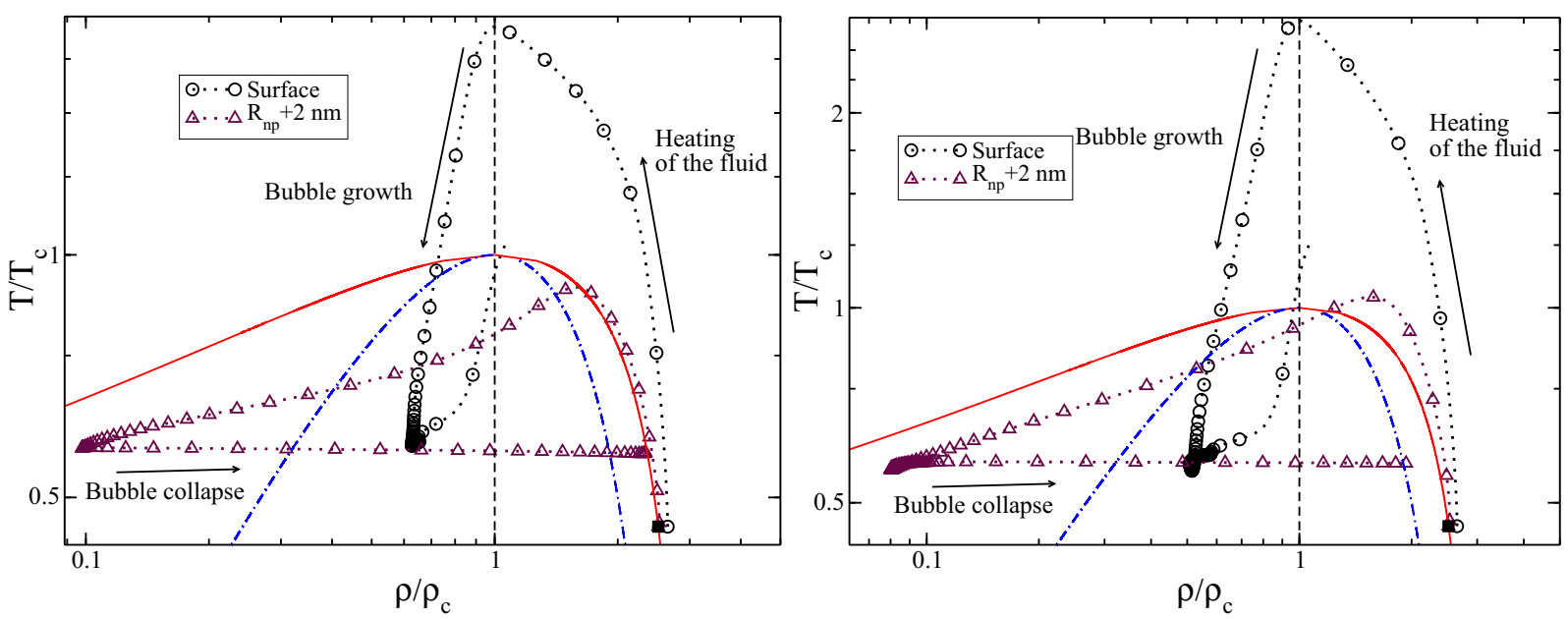

FIG. 11. (Color online) Temporal evolution of the system on a $T$ - $\rho$ diagram from the beginning of the pulse to the collapse of the first bubble, at the GNP surface (black circles) and $2 \mathrm{~nm}$ from the GNP surface (purple triangles). Here $R_{\mathrm{np}}=10 \mathrm{~nm}, F=162 \mathrm{~J} / \mathrm{m}^{2}$ (left), and $F=271 \mathrm{~J} / \mathrm{m}^{2}$ (right). The solid red line stands for the saturation curve, which delimits the stable and metastable phases for the vapor (on the left) and for the liquid (on the right). The dashed-dotted blue line stands for the spinodal curve, which represents the limit between the metastable phases and the unstable liquid-vapor zone (at the center). The black square on the right indicates the thermodynamic state of the system prior to heating $\left(\rho_{\infty}, \mathrm{T}_{\infty}\right)$.

In conclusion, we saw that the thermodynamic evolution of the vapor phase may be described by a bulklike treatment, at least as soon as locally the fluid has cooled down below the critical temperature $T_{c}$. This is mostly related to the change of the nature of the energy flux at the GNP surface when the fluid vaporizes, which concentrates the temperature gradient in the liquid, which locally follows the saturation line consistent with this gradient. These considerations shed light on the evolution of the pressure inside the nanobubble, which we analyze in the next section.

\section{PRESSURE}

As we briefly mentioned, the pressure in the fluid first increases drastically when a first vapor layer surrounds the GNP.

\section{A. Pressure wave}

This sudden increase is accompanied by the propagation of a pressure wave, as best seen in Fig. 12. Here we plot the thermodynamic pressure $P_{\mathrm{VdW}}$ profiles in the fluid for different times after vaporization sets in. Remarkably the pressure in the liquid close to the particle can reach very large values, well above the critical pressure. This is reasonable since the formation and further growth of a nanobubble requires a typical pressure on the order of the Laplace pressure $\Delta P_{\text {Laplace }}=2 \gamma / R_{\mathrm{np}} \simeq 10 \mathrm{MPa}$ for a nanoparticle of radius $R_{\mathrm{np}}=15 \mathrm{~nm}$. This value compares well with our simulation results when a bubble is generated: in the inset of Fig. 12 the pressure in the fluid close to the particle at $21.75 \mathrm{ps}$ is more than $40 \mathrm{MPa}$, which is well above the Laplace pressure.

While the bubble forms, a part of the excess pressure is released through a pressure wave that flows away from the particle. It is important to remark that the generation of a pressure wave is due to the sudden dilation of the liquid, and it is emitted before the nanobubble appears. In the case depicted in Fig. 12, the nanobubble forms at $50 \mathrm{ps,} \mathrm{and} \mathrm{the} \mathrm{pressure} \mathrm{wave}$ has already moved over approximately $70 \mathrm{~nm}$. The pressure wave moves into the liquid, with a velocity which is found to be close to the speed of sound in the unheated liquid. The mean value of the wave velocity has been determined based on the distance traveled by the maximum of the wave, as schematically represented in Fig. 12. The generation of this pressure wave is of great interest and is considered as one of the phenomena that lead to cell destruction in medical applications [13]. Our simulations confirm that though it is generated locally at the nanoparticle surface it moves over large

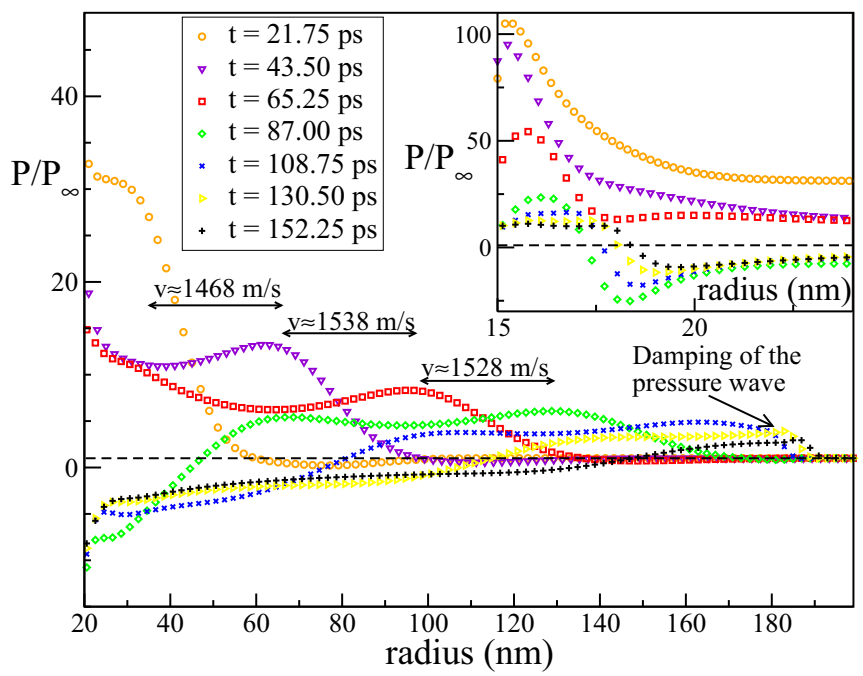

FIG. 12. (Color online) Pressure wave $P_{\mathrm{VdW}}$ profiles in the fluid surrounding a nanoparticle at different times. Here $R_{\mathrm{np}}=15 \mathrm{~nm}$ and $F=101.25 \mathrm{~J} / \mathrm{m}^{2}$. The dashed lines stand for the pressure $P_{\infty}$ far from the GNP. The inset is a closer view of the GNP surface. The arrows on the main figure indicate the relative displacement of the pressure maximum between two times used to calculate the corresponding average velocity of the pressure wave. 


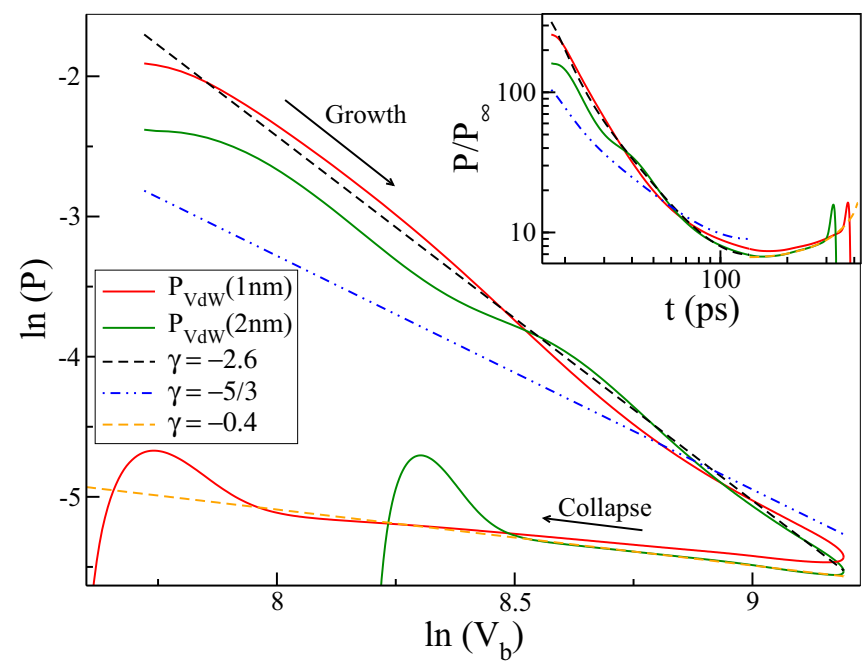

FIG. 13. (Color online) Evolution of the local pressure $P$ in the vapor bubble as a function of the nanobubble instantaneous volume $V_{b}$. The pressure is estimated at a distance 1 and $2 \mathrm{~nm}$ from the GNP surface. The dashed lines show the behavior $P \sim V^{\gamma}$, with $\gamma=-2.6,-5 / 3$, and -0.4 . The inset shows the evolution of the internal pressure with time. Here $R_{\mathrm{np}}=10 \mathrm{~nm}$ and $F=271 \mathrm{~J} / \mathrm{m}^{2}$.

distances and could therefore interact with the cell components far from the particle [34,35].

After the bubble generation (total time elapsed $t \geqslant 50 \mathrm{ps}$ in the case presented in Fig. 12) the pressure within the vapor becomes rapidly homogeneous and decreases during the bubble growth. On the inset of Fig. 12 we see that in the liquid close to the vapor $(r \geqslant 17 \mathrm{~nm})$ the pressure is negative, consistent with the local metastable nature of the liquid state.

\section{B. Internal nanobubble pressure}

To further elucidate the thermodynamic nature of the growth of the bubble, we plotted the evolution of the pressure inside the vapor as a function of the instantaneous bubble volume $V_{b}$ in Fig. 13. The simulation results are compared to power law behaviors: $P \sim V_{b}^{-\gamma} \cdot \gamma=1$ classically corresponds to an isothermal evolution, and $\gamma=5 / 3$ refers to an adiabatic evolution.

Based on Fig. 13 we conclude that indeed neither behavior is observed. The growth of the nanobubble is rather consistent with an exponent $\gamma=2.6$, while the collapse corresponds to $\gamma=0.4$. Indeed, these discrepancies may be explained, given the evolution of the number of vapor molecules during the lifetime of the nanobubble, as represented in Fig. 14. Clearly, the number of vapor molecules inside the nanobubble is not constant and is decreasing during both the bubble growth and collapse. Hence, condensation becomes operative as soon as a nanobubble is formed. This conclusion enables us to appraise the different sources of energy exchange in the nanobubble. Figure 15 summarizes the different contributions to the energy rate in the nanobubble and in the liquid. During the growth, dilation of the vapor occurs and is concomitant with condensation as evidenced by the strong local compression of the interface. Also, thermal conduction turns out to be negligible in the vapor bubble, while it is significant in the

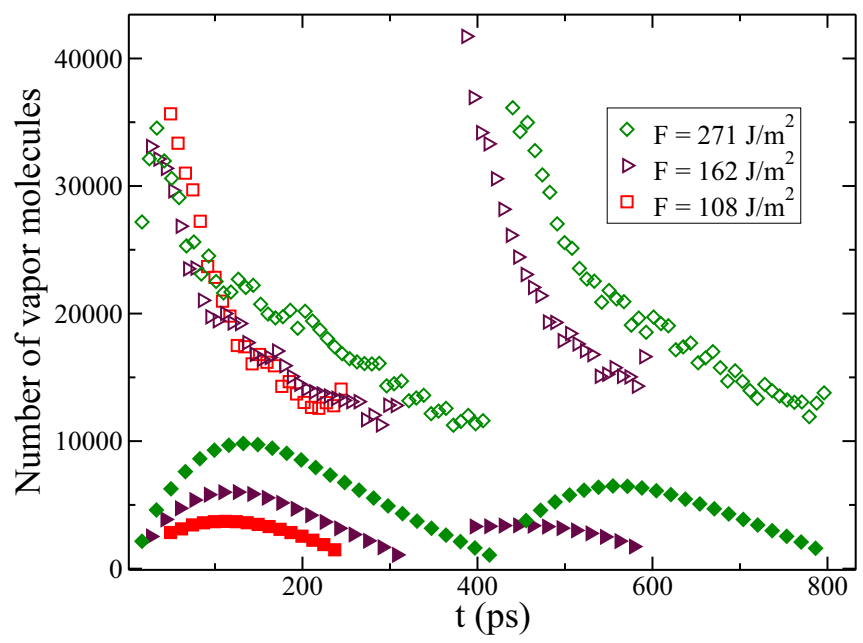

FIG. 14. (Color online) Evolution of the number of vapor molecules in the bubble, for different fluences (empty symbols). The corresponding volume bubbles are displayed with filled symbols. Here $R_{\mathrm{np}}=10 \mathrm{~nm}$.

liquid due to the finite temperature gradient. Viscous heating is small as compared with the other sources of heating, in the vapor and in the liquid as well. The behavior of the vapor phase is thus compatible with an adiabatic expansion since very little heat is exchanged, but the number of molecules in the vapor phase is not constant. During the collapse, compression in the vapor competes with thermal conduction to maintain the vapor temperature constant. Again, condensation sets in as represented by the peak of the compression term at the interface. Viscous heating contributes only slightly to heat the interface. However, if viscosity has little effect on thermal transport, we shall see below that it still plays an important role in momentum transport.

Alternately, the role of the viscous forces may be inferred from a mechanical analysis. We already showed that the evolution of the nanobubble radius may be described in terms of the Rayleigh-Plesset equation, which is classically used to describe cavitation dynamics [12]:

$$
\begin{gathered}
m \rho_{\text {liq }}\left(R_{b} \ddot{R}_{b}+\frac{3}{2} \dot{R}_{b}^{2}\right)=P_{i}(t)-P_{e}(t)-2 \frac{\gamma}{R_{b}}-4 \eta \frac{\dot{R}_{b}}{R_{b}}, \\
P_{i}(t)=P_{i}^{\max }\left[\frac{V_{b, \max }}{V_{b}(t)}\right]^{\zeta}
\end{gathered}
$$

where $R_{b}$ is the bubble radius, $P_{i}$ and $P_{e}$ are the internal and external pressures, $V_{b}(t)$ and $V_{b, \max }$ are the nanobubble volumes at time $t$ and when the bubble radius is maximal, and $\gamma$ is the fluid surface tension. $m \rho_{\text {liq }}$ is the mass density of the liquid far from the nanoparticle given in Table I. The viscosity $\eta$ used here is the shear viscosity of water [36].

We have compared in Fig. 16 the relative contribution of the different mechanical driving forces contained in the Rayleigh-Plesset equation (21). Of particular interest here is the relaxation of the internal pressure, which turns out to be very large during the bubble growth. At the early stage of the growth, the difference in pressure between the internal 

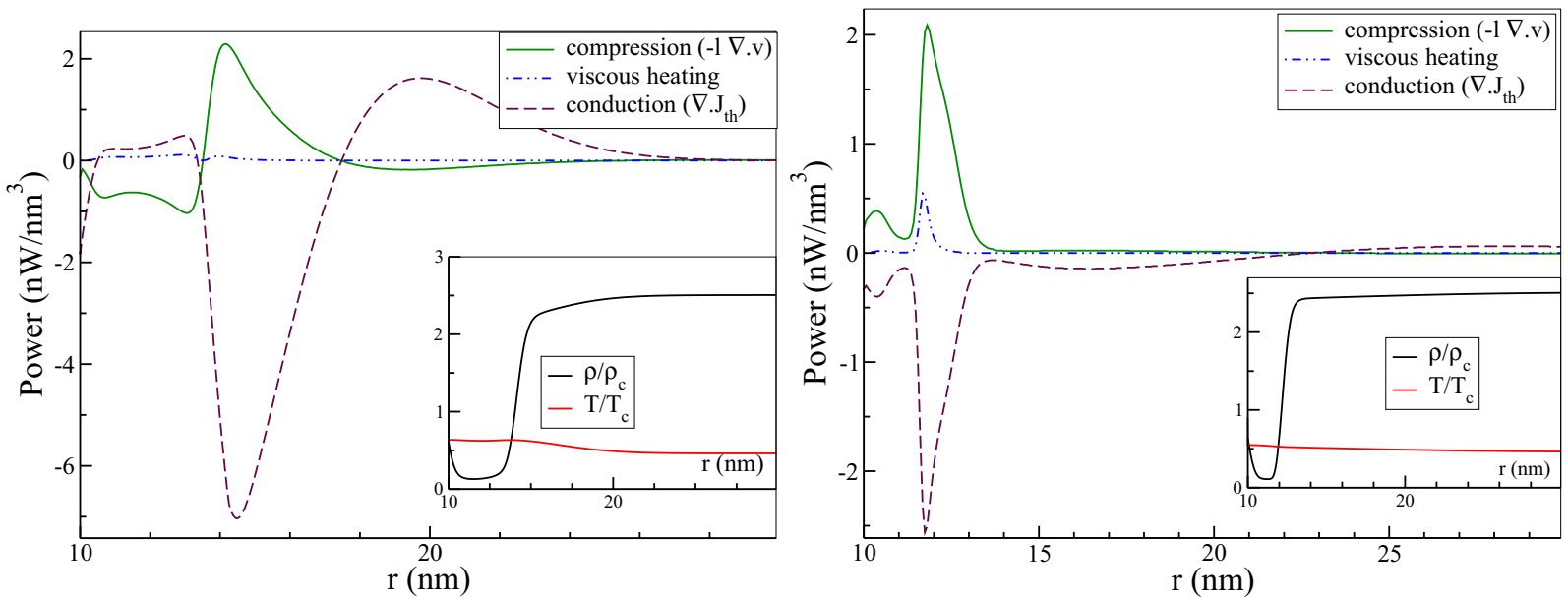

FIG. 15. (Color online) Spatial profiles of energy rates (per unit of volume) during bubble growth (left) and collapse (right) (compression $-l \nabla \cdot v$; conduction $-\nabla \cdot J_{\text {th }}$ with $J_{\text {th }}=-\lambda \nabla T$; and viscous heating). The inset shows the corresponding density and temperature profiles. Here $R_{\mathrm{np}}=10 \mathrm{~nm}$, the fluence is $271 \mathrm{~J} / \mathrm{m}^{2}$, and the ballistic flux is set at zero.

and the external pressure overcomes the Laplace pressure. The mechanical analysis allows us to understand how the nanobubble may accommodate very large internal pressures.

We can evaluate the relative importance of the different terms through the computation of the associated energy during the growth. The results are given in Table II. When the bubble reaches its maximum radius, the energy provided by the difference in pressure between the vapor and the surrounding liquid is balanced by the work of the Laplace pressure. More importantly, the viscous forces dissipate energy in a more important way than any other contribution. This importance of the viscosity can be appreciated by the estimation of the Reynolds number during the bubble growth:

$$
\begin{aligned}
\mathcal{R} e & =\frac{m \rho_{\text {liq }} v_{\text {growth }}\left(R_{\max }-R_{\mathrm{np}}\right)}{\eta} \\
& =\frac{m \rho_{\text {liq }}\left(R_{\text {max }}-R_{\mathrm{np}}\right)^{2}}{\eta \Delta t_{\text {growth }}},
\end{aligned}
$$

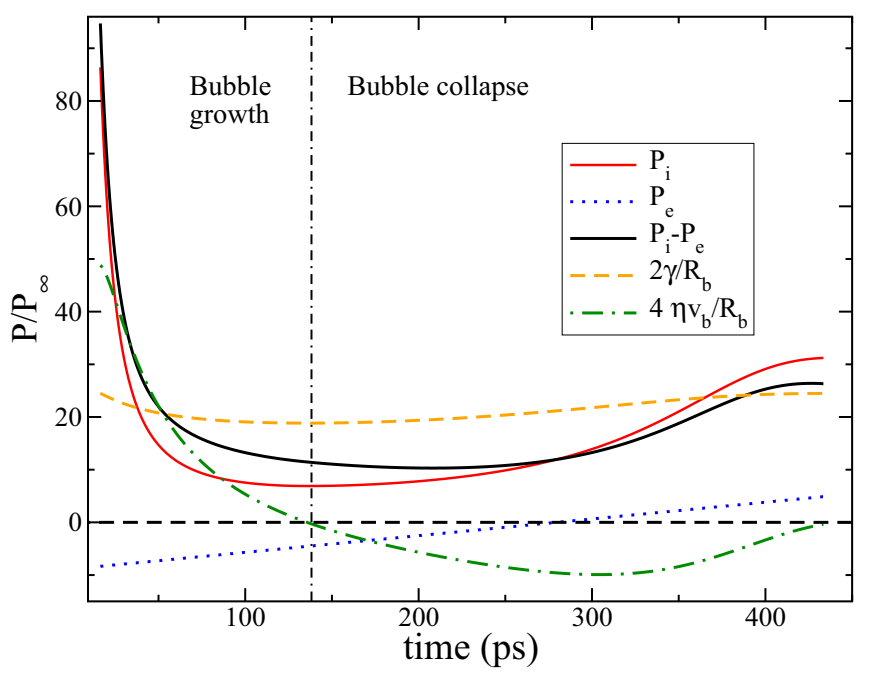

FIG. 16. (Color online) Temporal evolution of the terms driving the bubble dynamics in the Rayleigh-Plesset equation (21). $v_{b}$ stands for the liquid-vapor interface velocity. where $v_{\text {growth }}$ is the average velocity of the bubble during its growth. An evaluation of this Reynolds number gives 0.15 for the case $F=162 \mathrm{~J} / \mathrm{m}^{2}$ and 0.2 for $F=271 \mathrm{~J} / \mathrm{m}^{2}$, which confirms the importance of the viscous processes. The same calculation for the collapse gives 0.07 and 0.1 , respectively, which highlights the effect of viscosity on momentum transport.

\section{CONCLUSION}

In summary, we presented a thermodynamic analysis of the dynamics of vapor nanobubbles in water, based on a phasefield hydrodynamics model. This situation is relevant to recent experiments, where the vapor nanobubbles have been generated by metallic nanoparticles heated by a strong laser pulse.

We emphasize that the nanobubble is generated under a very large temperature gradient, and locally exceeding the spinodal temperature. In a few picoseconds after its generation however, the temperature inside the bubble sharply drops off by hundredths of kelvins. This fast relaxation is attributed to the sudden increase of the interfacial thermal resistance, present at the nanoparticle-water interface. After its generation, the temperature inside the bubble becomes homogeneous, while a finite temperature gradient is maintained in the liquid due to the ballistic heat flux flowing across the bubble. The description of the nanobubble as a quasihomogeneous medium allows us to unambiguously identify the thermodynamic path followed by the vapor. The thermodynamics analysis confirms our previous finding [12]: the bubble expansion is found to be adiabatic, although the number of molecules in the vapor phase is not constant and corresponds to fast vapor cooling, while the

TABLE II. Energy $\left(10^{-2} \mathrm{fJ}\right)$ associated with the different terms driving the bubble growth in Eq. (21) for $F=271 \mathrm{~J} / \mathrm{m}^{2}$.

Vapor pressure External pressure Pressure jump Laplace Viscosity

\begin{tabular}{lcccc}
$P_{i}$ & $P_{e}$ & $P_{i}-P_{e}$ & $2 \gamma / R_{b}$ & $4 \eta \dot{R}_{b} / R_{b}$ \\
\hline 5.25 & -2.81 & 8.06 & -8.29 & -9.68 \\
\hline
\end{tabular}


bubble collapse is rather found to be isothermal. This relative asymmetry also found experimentally is interpreted by the role played by viscous forces in the nanobubble dynamics. At the early stage, the pressure inside the bubble is larger than the Laplace pressure, which is huge at the scale considered.
The excess pressure is relaxed by the viscous stress developed by the liquid until the bubble becomes mature and collapse proceeds.

In the future, we plan to study how the nanobubble maximal size may be optimized.
[1] Z. Liang, K. Sasikumar, and P. Keblinski, Phys. Rev. Lett. 111, 225701 (2013).

[2] H.-R. Jiang, H. Wada, N. Yoshinaga, and M. Sano, Phys. Rev. Lett. 102, 208301 (2009).

[3] M. Yang and M. Ripoll, Soft Matter 10, 1006 (2014).

[4] S. Merabia, J.-L. Barrat, and L. Lewis, J. Chem. Phys. 134, 234707 (2011)

[5] D. Rings, R. Schachoff, M. Selmke, F. Cichos, and K. Kroy, Phys. Rev. Lett. 105, 090604 (2010).

[6] L. Joly, S. Merabia, and J.-L. Barrat, Europhys. Lett. 94, 500007 (2011).

[7] A. Siems, S. A. L. Weber, J. Boneberg, and A. Plech, New J. Phys. 13, 043018 (2011).

[8] O. Neumann, C. Feronti, A. D. Neumann, A. Dong, K. Schell, B. Lu, E. Kim, M. Quinn, S. Thompson, N. Grady, P. Nordlander, M. Oden, and N. J. Halas, Proc. Natl. Acad. Sci. USA 110, 11677 (2013).

[9] E. Y. Lukianova-Hleb and D. O. Lapotko, Appl. Phys. Lett. 101, 264102 (2012).

[10] K. Saskumar and P. Keblinski, J. Chem. Phys. 141, 234508 (2014).

[11] A. Polman, ACS Nano 7, 15 (2013).

[12] J. Lombard, T. Biben, and S. Merabia, Phys. Rev. Lett. 112, 105701 (2014).

[13] D. Lapotko, Cancers 3, 802 (2011).

[14] S. Merabia, S. Shenoguin, L. Joly, P. Keblinski, and J.-L. Barrat, Proc. Natl. Acad. Sci. USA 106, 15113 (2009).

[15] Z. B. Ge, D. G. Cahill, and P. V. Braun, Phys. Rev. Lett. 96, 186101 (2006).

[16] N. Shenogina, R. Godawat, P. Keblinski, and S. Garde, Phys. Rev. Lett. 102, 156101 (2009).

[17] A. Pham, M. Barisik, and B. Kim, J. Chem. Phys. 139, 244702 (2013).

[18] S. Merabia, P. Keblinski, L. Joly, L. J. Lewis, and J.-L. Barrat, Phys. Rev. E 79, 021404 (2009).
[19] R. Teshigawara and A. Onuki, Phys. Rev. E 82, 021603 (2010).

[20] A. Onuki, Phys. Rev. E 75, 036304 (2007).

[21] K. Schweizer and E. J. Saltzman, J. Phys. Chem. B 108, 19729 (2004).

[22] http://webbook.nist.gov/chemistry/fluid/

[23] P. G. de Gennes, Rev. Mod. Phys. 57, 827 (1985).

[24] G. Baffou and H. Rigneault, Phys. Rev. B 84, 035415 (2011).

[25] V. Juve, M. Scardamaglia, P. Maioli, A. Crut, S. Merabia, L. Joly, N. Del Fatti, and F. Vallée, Phys. Rev. B 80, 195406 (2009).

[26] E. T. Swartz and R. O. Pohl, Rev. Mod. Phys. 61, 605 (1989).

[27] J.-L. Barrat and F. Chiaruttini, Mol. Phys. 101, 1605 (2003).

[28] D. G. Cahill, W. K. Ford, K. E. Goodson, G. D. Mahan, A. Majumdar, H. J. Maris, R. Merlin, and S. R. Philpot, J. Appl. Phys. 93, 793 (2003).

[29] Z. Liang, W. Evans, and P. Keblinski, Phys. Rev. E 87, 022119 (2013).

[30] M. Bond and H. Struchtrup, Phys. Rev. E 70, 061605 (2004).

[31] J. R. T. Seddon, H. J. W. Zandvliet, and D. Lohse, Phys. Rev. Lett. 107, 116101 (2011).

[32] F. Q. Hu, X. D. Li, and D. K. Lin, J. Comp. Phys. 227, 4398 (2008).

[33] F. D. Hastings, J. B. Schneider, and S. L. Broschat, J. Acoust. Soc. Am. 100, 3061 (1996).

[34] L. Hou, M. Yorulmaz, N. R. Verhart, and M. Orrit, New J. Phys. 17, 013050 (2015).

[35] The use of PMLs at the boundaries prevents the pressure wave from flowing back toward the nanobubble, which would lead to its premature collapse. Simulations run without those PMLs confirmed this premature collapse. Running simulations using a box 10 times larger without damping conditions led to the same bubble dynamics as presented here, which confirms that the PMLs do not affect our results.

[36] A. Prosperetti and M. S. Plesset, J. Fluid Mech. 85, 349 (1978). 